CONF-900143--30

DE9 0007797

\title{
SEQUENTIAL PROBABILITY RATIO TESTS FOR REACTOR SIGNAL VALIDATION AND SENSOR SURVEILLANCE APPLICATIONS
}

\author{
by \\ Keith Humenik \\ University of Maryland Baltimore County \\ Baltimore, Maryland 21228 \\ and \\ Kenny C. Gross \\ Argonne National Laboratory \\ Argonne, Illinois 60439
}

Address for Correspondence and Return of Proofs

Keith Humenik, Computer Science Department, University of Maryland Baltimore County, Baltimore, Maryland 2122S

27 Pages, 1 Table, 3 Figures

November 9, 1989
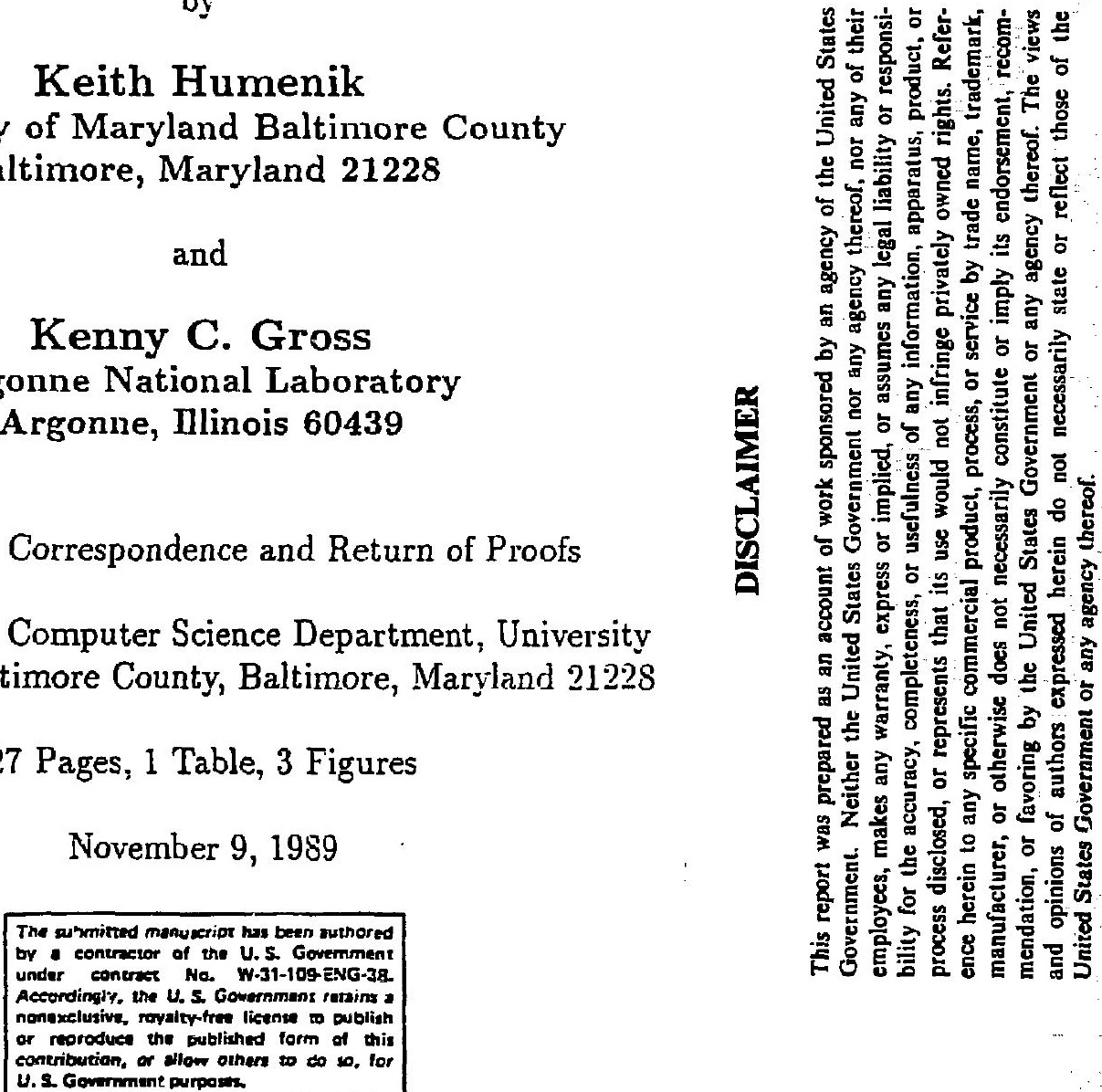

Work supported by the U.S. Department of Energy, Civilian Reactor Development, under Contract W-31-109-ENG-38 


\begin{abstract}
This paper examines the properties of sequential probability ratio tests (SPRT's) and the application of these tests to nuclear power reactor operation. Recently SPRT's have been applied to delayedneutron (DN) signal data analysis using actual reactor data from the Experimental Breeder Reactor-II, which is operated by Argonne National Laboratory. The implementation of this research as part of an expert system is described. Mathematical properties of the SPRT are investigated, and theoretical results are validated with tests that use DN-signal data taken from the EBR-II in Idaho. Variations of the basic SPRT and applications to general signal validation are also explored.
\end{abstract}




\section{Introduction}

A sequential probability ratio test (SPRT) is a statistical hypothesis test which differs from the standard fixed-sample test in the way in which statistical observations are empioyed. In the fixed-sample test a given number of observations are used to select one hypothesis from two or more alternatives. The SPRT, however, examines one observation at a time and at some point makes a decision and selects an hypothesis, or, if the SPRT is not closed, it may continue to examine observations indefinitely without reaching a conclusion with some nonzero probability. SPRT's have been studied extensively in the statistical literature for quite some time beginning with Wald [1] and Wald and Wolfowitz [2]. Only more recently, however, have SPRT's found beneficial applications in the areas of safety and reliability of nuclear power reactor operation.

Given certain reasonable assumptions SPRT's have been shown to possess optimal properties versus corresponding fixed-sample tests $[2,3,4]$. For example, when choosing between two alternative hypotheses, the SPRT can malie a correct decirion faster (on average) than the fixed-sample test to which it corresponds. When this is the 
case it is said that the SPRT is more efficient than the fixed-sample test. The validity of the above statement in a specific instance depends upon a number of statistical properties of the problem being studied, such as the form of the hypotheses, the underlying statistical distribution, the true value of various parameters and the specific kind of SPRT being used.

More recently SPRT's have been applied to pattern recognition problems [3]. A compound SPRT has been used to improve efficiency in two-class recognition problems with first-order Markov dependence among pattern classes [5], and a nonparametric SPRT has been formed to investigate Markov dependent data and problems which test more than two hypotheses [6].

It has been shown that the SPRT is generally more efficient than the fixed-sample size likelihood ratio test for the problem of detecting a constant signal in additive noise [7]. The SPRT has also been used to estimate system availability in systems which are continuously "on station", eg. early warning systems, patrol craft, etc. [8] A project was initiated at the U.S. Nuclear Regulatory Commission in 1980 to attempt to transform the SPRT into a practical 
tool for nuclear power plant data analyses [9]. This study endorses the value of a SPRT and encourages further testing. A truncated SPRT has been shown to possess large potential savings in a:erage time spent monitoring employees for radiation exposure at the Rocky Flats Plant in Golden, Colorado [10]. A SPRT has also been suggested for rapid decision making and the ability to track signals anitted by radiation monitors used for nuclear safeguards and security [11].

The research presented in this paper primarily consists of the development of new SPRT signal-analysis tools and focuses on the integration of these tools into an AI based expert system to be used as an operator decision aid for liquid-metal cooled nuclear reactors (LMR's) that are licensed to operate with failed fuel [1?]. The expert system is embodied in a device which monitors, processes and interprets information from nine groups of redundant plant sensors and displays to the reactor operator the diagnostic information needed to make proper decisions about technical-specification conformance during run-beyond-clad-breach (RBCB) operation. 
The expert system is expected to enhance the safety, economics and licensibility of future-design LMR's. The system will enhance plant safety by making available to the operator online diagnosis and interpretation of a bewildering variety of interacting physical variables during exposed-fuel operation. Currently, full interpretation of these variables requires several days to weeks of detailed analysis by teams of specialists. The new system will provide the operator with very rapid (within one minute) identification of off-normal $\mathrm{RBCB}$ conditions, thereby enabling him to terninate or avoid events which might challenge safety or radiological performance guidelines. The system will enhance plant availability and economics by minimizing unnecessary reactor trips caused by events having no safety signifcance.

A detailed mathematical analysis has been performed on the applications of SPRT's to RBCB operation in relation to the aforementioned expert system. Current practice in all countries that have LMR programs (except possibly the Soriets) is to set conservative shutdown limits on the magnitude of delayed-neutron (DN) signals coming from breached fuel. By combining and evaluating informa- 
tion from several groups of redundant plant sensors, the AI-based system will make it possible to significantly relax the conservatism in DN shutdown limits without compromising plant-safety assurance. Currently, the SPRT is being applied to the problem of providing rapid annunciation of discrepant signals or failed sensors with minimal "false alarm" probabilities. Since DN signals are functions of a number of interacting physical variables, a future goal is to apply the SPRT to questions about individual physical variables and combinations of physical variables influencing the DN signal. Examples include:

1. determining whether a change in the statistical quality of a DN signal (annunciated with a SPRT developed in the present paper) is a result of a change in source condition (breach condition or fission rate), cr a change in primary pump performance characteristics and

2. using a modification of the SPRT developed here for rapid departure-from-background annunciation, which can pinpoint the onset of a new breach in the presence of background DN events 
(from fissioning of "tramp" uranium) with greater accuracy and

a lower "false-alarm" probability than simple threshold-limit tests currentiy in use for LMR's.

In most cases, the SPRT will provide an answer to each of these questions faster than all other statistical tests.

The expert system described above has been developed for incorporation into the conceptual design of Argonne National Laboratory's Integral Fast Reactor. Plans are underway to install a prototype apparatus at the EBR-II in Idaho. The device is also being incorporated into the conceptual design of General Electric's Power Reactor Inherently Safe Module.

\section{Preliminaries}

Consider the problem of discriminating between two simple hypotheses

$$
H_{0}: \theta=\theta_{0}, \quad H_{1}: \theta=\theta_{1}, \quad \theta_{0} \neq \theta_{1}
$$

In this case, we concentrate on the family $F_{n}\left(X_{n} ; \theta\right), \theta=\theta_{0}, \theta_{1}$ (ie. the cumulative distribution function of the observations $x_{1}, x_{2}, \ldots, x_{n}$ given the parameter $\theta$ ) containing only two distinct distributions [4]. 
In the usual sense, define

$\alpha=$ the probability that $H_{1}$ is chosen or accepted when $H_{0}$ is true,

$1-\alpha=$ the probability that $H_{0}$ is accepted when $H_{0}$ is true, $\beta=$ the probability that $H_{0}$ is accepted when $H_{1}$ is true; $1-\beta=$ the probability that $H_{1}$ is accepted when $H_{1}$ is true, and $\mathrm{n}=$ the number of observations required to accept either $H_{0}$ or $H_{1}$.

$\alpha$ and $\beta$ are called error probabilities and are usually determined by the nature of the problem, and $\mathrm{n}$ is called the sample number. There exist a number of statistical tests to approach this problem. A 'good' test should accept $H_{0}$ when $\theta$ is close to $\theta_{0}$ and reject $H_{0}$ (accept $H_{1}$ ) when $\theta$ is close to $\theta_{1}$ with high probability. Define the operating characteristic function $(\mathrm{OC}), Q(\theta)$, as the probability that $H_{0}$ is accepted as a function of $\theta$, and the power function, $P(\theta)$, as the probability that $H_{1}$ is accepted as a function of $\theta$. As in [4], we impose the validity criteria $P(n<\infty \mid \theta)=1$ for $\theta=\theta_{0}, \theta_{1}$, and

$$
Q\left(\theta_{0}\right) \geq 1-\alpha, \quad Q\left(\theta_{1}\right) \leq \beta
$$


Thus, the statistical test will always make a decision given a finite number of observations when $\theta=\theta_{0}$ or $\theta=\theta_{1}$. Also, the test will correctly accept $H_{0}$ when $\theta=\theta_{0}$ at least $100(1-\alpha)$ per cent of the time and will correctly accept $H_{1}$ when $\theta=\theta_{1}$ at least $100(1-\beta)$ per cent of the time. Note that if $P(n<\infty \mid \theta)=1$ for all values of $\theta$, the test is closed, i.e. it always makes a decision after some finite number of observations, and $Q(\theta)+P(\theta)=1$ for all $\theta$.

We now discuss two remaining questions in turn. Firstly, what statistical test shall we employ? A fixed-sample test, in which a constant $\mathrm{n}$ is selected so as to satisfy (2), is standard and simple. However, we shall choose a sequential probability ratio test for the reason discussed in the next paragraph. A sequential probability ratio test (SPRT) S(b,a) for (1) is defined by [4]: Observe the sequence of observations $\left\{x_{i}\right\}(i=1,2, \ldots)$ successively, and at stage $n \geq 1$

(i) accept $H_{0}$ if $Z_{\pi} \leq b$,

(ii) reject $H_{0}$ if $Z_{n} \geq a$,

(iii) continue by observing $x_{n+1}$ if $b<Z_{n}<a$,

where the stopping bounds $(\mathrm{b}, \mathrm{a}),-\infty<b<a<\infty$, are two real 
numbers, and

$$
Z_{n}=\ln \frac{f_{n}\left(X_{n} ; \theta_{1}\right)}{f_{n}\left(X_{n} ; \theta_{0}\right)} \text { for } n \geq 1
$$

is the natural logarithm of the probability ratio at stage $\mathrm{n}$. The inequality $b<Z_{n}<a$ is called the critical incquwity of $v(\mathrm{~b}, \mathrm{a})$ at stage $\mathrm{n}$. The sample number $\mathrm{n}$ at which a decision is made depends upon the observations themselves and the value of $\theta$. In many cases, the average sample number (ASN) can be computed as a function of $\theta$ and is denoted by $E(n ; \theta)$.

It can be shown [4] that if $\mathrm{S}(\mathrm{b}, \mathrm{a})$ is any SPRT which tests $H_{0}$ vs. $H_{1}$ in (1) in a one-parameter family with $\mathrm{OC}$ function $Q(\theta)$ and ASN function $E(n ; \theta)$, if the $x_{i}$ are independent identically distributed random variables and if certain other quite general assumptions hold, then for any other rival statistical test $\mathrm{S}$ whose OC function satisfies $Q_{S}(\theta) \geq Q(\theta)$ for all $\theta<\theta^{\prime}, Q_{S}(\theta) \leq Q(\theta)$ for all $\theta>\theta^{\prime}$, where $\theta^{\prime}$ satisfies $E\left(z ; \theta^{\prime}\right)=0$ and

$$
z=\ln \frac{f\left(x ; \theta_{1}\right)}{f\left(x ; \theta_{0}\right)}
$$

we have $E_{S}(n ; \theta) \geq E(n ; \theta)$ for all $\theta \neq \theta^{\prime}$. Note that $E(z ; \theta)$ is the expected value of $z$ and is, in general, a function of $\theta$, since $z$ is 
a function of $x$, and $x$ is a function of $\theta \cdot \theta^{\prime}$ is the value of $\theta$ that makes $E(z ; \theta)$ equal to zero. The proof that $E_{S}(n ; \theta) \geq E(n ; \theta)$ for all $\theta \neq \theta^{\prime}$ is not given here, but is provided in detail in [4]. Hence, the SPRT makes a faster decision (on average) than any other test, which has equal or smaller error probabilities, provided the above assumptions are satisfied. Such a test is called a uniformly $m$-st efficient (UME) test. This is the major reason why the SPRT is a useful test and, in many cases, the best statistical test.

\section{Main Results}

We now turn to the second question of interest. What problem do we wish to solve? The problem of interest is that of determining whether DN detectors are functioning correctly. Consider the case in which there are two redundant detectors monitoring the same process. (See Figure 1.)

We form a discrete difference function $X_{k}=X_{1}\left(t_{k}\right)-X_{2}\left(t_{k}\right)$ by subtracting corresponding obsezvations of $X_{1}$ and $X_{2}$ sampled at times $t_{k}, k=1,2, \ldots$. Since both sensors are monitoring the same process, $X_{k}$ should have mean 0. (See Figure 2.) We develop the 
$X_{1}$

Sensor \#1

Time

$X_{1}=$ signal from Sensor $\# 1$

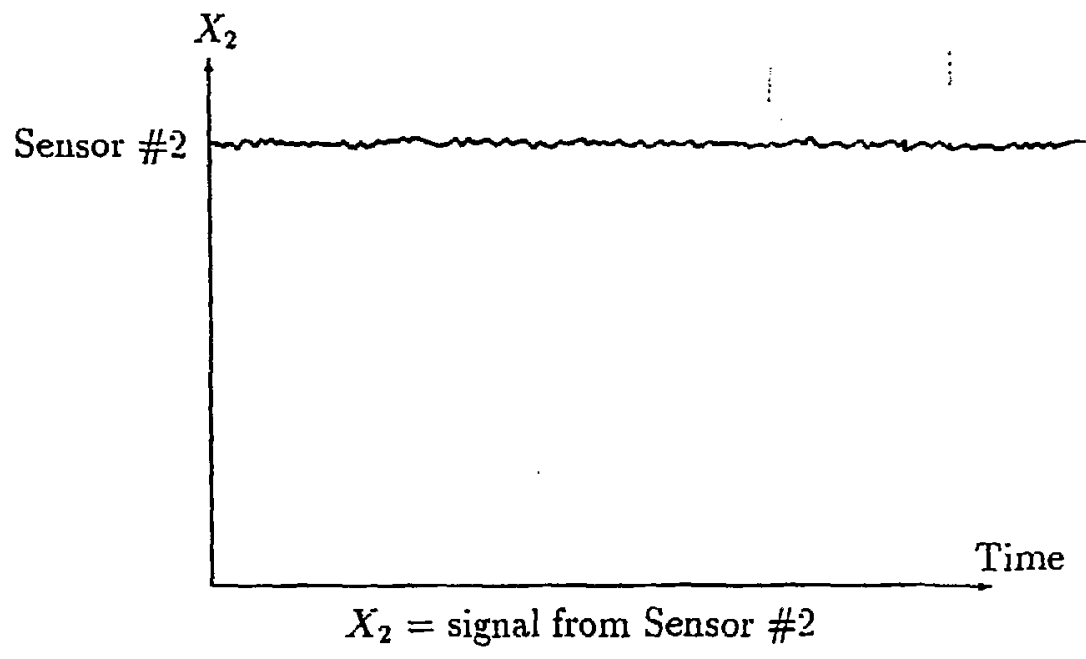

Figure 1. Two detectors monitoring the same process. 


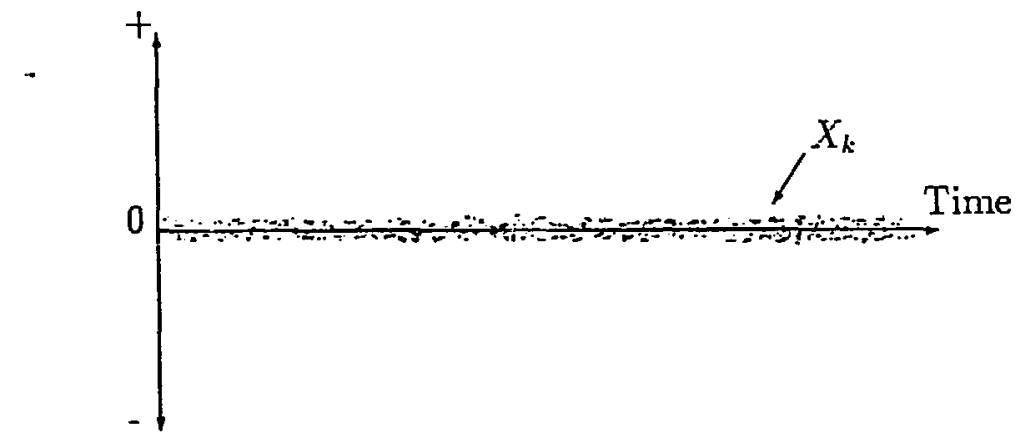

Figure 2. Graph of the difference function $X_{k}$.

following statistical hypotheses and use a SPRT to test them. $H_{0}: X$ is Gaussian with mean $\mu_{0}=0$ and variance $\sigma^{2}$ $H_{1}: X$ is Gaussian with mean $\mu_{1} \neq 0$ and variance $\sigma^{2}$

The value of $\sigma^{2}$ can be estimated from actual data. If the SPRT accepts $H_{1}$, we declare sensor 1 or 2 failed. Note, however, that the SPRT alone does not indicate which sensor has failed.

If one sensor fails, it is possible for both $\mu$ and $\sigma^{2}$ to change. However, the SPRT as defined herein only tests for a change in the mean. Although the SPRT will continue to accept the null hypothesis, $H_{0}$, if only the variance of the signal changes, the SPRT is sensitive to a change in the variance of the signal. Because the ASN is directly proportional to $\sigma^{2}$, the variance of the difference function, as shown below, a change in the variance of one signal will 
affect $\sigma^{2}$ and will subsequently cause a change in the ASN of the SPRT. Further study is currently underway to examine the effects of various kinds of failures on the properties of the SPRT.

Our test has been formulated as a test of Gaussian means. This leads to the question of whether the underlying DN sigal distribution is Gaussian. This question has been explored using actual DN signals from experiments conducted at full power in EBR-II. Although a number of statistical tests of normality, including the sensitive Shapiro-Wilk test [13], indicate that the underlying distribution of $X$ is not Gaussian, it can be seen (See Table 1) that the assumption that $X$ is normally distributed does not significantly affect the performance of the SPRT for our application. That is, for the simulated SPRT applied to real DN-signal data the ASN was seldom more than $10 \%$ greater and was often significantly iess than the theoretically predicted value of the ASN using the assumption that the DN-signal data were normally distributed. Hence, the mathematical theory of the SPRT applied to testing the mean of a Gaussian distribution provides accurate and conservative estimates for the ASN of the SPRT that has been applied to real DN-signal 
data. This is fortunate, since much theoretical work has already been done in the area of SPRT's applied to normally distributed data.

The question of how close to Gaussian the signal has to be is very complicated. This issue is being studied carefully for DN signals, as well as for pump power and pump speed signals, and it has been shown that, in general, the SPRT becomes less accurate when $\left|\mu_{1}-\mu_{0}\right|<\sigma$. For this reason, the authors are studying the applicability of various transformations to normality. SPRT's can be formulated for applications to signals contaminated by nonGaussian noise, as long as the underlying signal distribution can be represented mathematically in some closed form or transformed to normality, but the analytical complexity may become far groater.

Signals containing periodic components pose no problem to SPRT analysis provided the components are in phase on the two instrument channels, as is the case when the periodicities originate in the physical process being observed by redundant sensors with identical time constants. In this case the periodic phenomena are filtered by 
the differencing process.

Several properties of the SPRT are of interest. These are:

1. Reliability

2. Average sample number

3. Effect of the choice of $\mu_{1}$

4. Robustness

The reliability or accuracy of the SPRT is determined by the error probabilities, $\alpha$ and $\beta$. Since these values are generally chosen based upon the nature of the problem, they can be selected by the SPRT user. Às expected, their values do have an effect on other properties of the SPRT as shown below.

The authors have determined various other facts regarding the remaining SPRT properties. It can be shown [4] that the ASN is given by the following approximation when the underlying distribution is Gaussian:

$$
E[n ; \mu] \approx \frac{2 \sigma^{2}\left\{\frac{A^{h}-1}{A^{h}-B^{h}} \ln \frac{\beta}{1-\alpha}+\frac{1-B^{h}}{A^{h}-B^{h}} \ln \frac{1-\beta}{\alpha}\right\}}{\left(\mu_{1}-\mu_{0}\right)\left(2 \mu-\mu_{1}-\mu_{0}\right)}
$$

where

$$
h=\frac{\mu_{1}+\mu_{0}-2 \mu}{\mu_{1}-\mu_{0}}, A=\frac{1-\beta}{\alpha}, B=\frac{\beta}{1-\alpha}
$$

and $\ln$ is the natural logarithm. Clearly, the ASN is a function of 
$\mu$, the true mean of the underlying distribution. As can be seen in Table 1, this approximation can be used to provide an accurate theoretical estimate of the ASN when the SPRT is applied to real DN-signal data. Note that the approximate ASN:

1. is directly proportional to $\sigma^{2}$

2 . is inversely proportional to $\left(\mu_{1}-\mu_{0}\right)^{2}$

3 . is bounded, ie. has a maximum value

4. increases slowly as $\alpha$ and $\beta$ decrease

It is not surprising that the $\mathrm{ASN}$ increases as $\sigma^{2}$ does. One would expect the SPRT to take lcnger to make a decision when the values observed vary to a greater degree. Conversely, it is easier for the SPRT to select an hypothesis as $\mu_{1}$ and $\mu_{0}$ grow farther apart. In fact, the SPRT takes longest to make a decision when $\mu=\left(\mu_{1}+\mu_{0}\right) / 2$. The ASN reaches a maximum at this point. Finally, a decrease of one order of magnitude in $\alpha$ and $\beta$ only approximately doubles the ASN. This is what we mean by 'increases slowly.'

The above properties of the ASN are encouraging. The SPRT also appears to be robust with respect to a number of important probability distributions. Hence, even if the underlying distribution 
is not the distribution being used in theoretical calculations, those calculations can still often be used as an accurate estimate of the SPRT properties, as was seen to be the case in our research on the $\mathrm{DN}$-signal data. What we desire is an underlying distribution, which, although it may not statistically be Gaussian, Poisson, etc., is 'close enough' for our theory to provide reasonable estimates. A distribution is close enough if

$$
\frac{s-t}{t} \leq p
$$

where $s$ is the simulated value, $t$ is the theoretical value, and $0<p<1$ is the allowable percentage error between the theoretical and actual OC, power and ASN. This percentage error is determined by the application. The theoretical value is that theoretical value calculated under the assumption of Gaussian data.

These considerations lead us to a discussion of the third property of the SPRT. What value should be chosen for $\mu_{1}$. In general, the authors suggest choosing the value for $\mu_{1}$ based upon the sensitive malfunction value of $\mu$, say $\mu^{\prime}$. If the value of $\mu_{1}$ is chosen to be $4 \mu^{\prime}$, then the SPRT will begin signaling a problem (accepting $H_{1}$ ) relatively often when $\mu=\mu^{\prime}=\left(\mu_{0}+\mu_{1}\right) / 4$. However, it will do so 
quickly, since $\mu$ has not yet reached $\left(\mu_{0}+\mu_{1}\right) / 2$, the point at which the ASN is maximum. The value of $\mu^{\prime}$ depends upon the situation being ana!yzed and upon the nature of sensor malfunction, ie. linear, quadratic, step, exponential, etc. The value of $\sigma^{2}$ also has an effect on the choice of $\mu_{1}$ : since the ASN and robustness of the SPRT are functions of $\left(\mu_{1}-\mu_{0}\right)_{\prime}^{\prime} \sigma$. Additional work needs to be done in the area of how to choose $\mu_{1}$ appropriately.

\section{Example and Numerical Results}

Table 1 illustrates the ASN, OC and porrer functions of the SPRT for theoretical (assuming an underlying normal distribution) and simulated (using actual DN-signal data from EBR-II) tests. The critical inequality is

$$
\frac{\left(\ln \frac{\beta}{1-\alpha}\right) \sigma^{2}}{\mu_{1}-\mu_{0}}+\frac{\mu_{0}+\mu_{1}}{2} n<\sum_{i=1}^{n} x_{i}<\frac{\left(\ln \frac{1-\beta}{\alpha}\right) \sigma^{2}}{\mu_{1}-\mu_{0}}+\frac{\mu_{0}+\mu_{1}}{2} n
$$

Other values are $\mu_{0}=0, \sigma^{2}=800.5138, \alpha=\beta=0.001$, and $N=700$ data values. The three values of $\mu_{1}$ were chosen to be approximately equal to $\sigma\left(\mu_{1}=28\right), \frac{1}{2} \sigma\left(\mu_{1}=14\right)$, and $\frac{1}{5} \sigma\left(\mu_{1}=6\right)$. It should be noted that, in practice, six sensor readings per second are common. 
Thus an ASN value of 100 ind : thes that the SPRT takes approximately 17 seronus to select an hypothesis. This is much faster than methods currently used, which often take two to ten minutes. It can be seen that the theoretical values yield very accurate estimates for real DN-signal data. Errors are usually highest near $\mu=\left(\mu_{0}+\mu_{1}\right) / 2$, and they are conservative, ie. the theoretical ASN is higher than the simulated ASN. Corresponding fixed sample tests yield sample numbers of $\mathrm{n}=39\left(\mu_{1}=28\right), \mathrm{n}=156\left(\mu_{1}=14\right)$, and $\mathrm{n}=850\left(\mu_{1}=6\right)$, which are also greater than SPRT ASN's, even for simulations when $\mu$ is very close to $\left(\mu_{0}+\mu_{1}\right) / 2$. Discrepancies in the $\mathrm{OC}$ and power functions are also small, and indications are that the SPRT that is simulated on DN data is slightly more prone to signal a problem than is theoretically predicted. If this is a serious problem, $\alpha$ and $\beta$ can be appropriately decreased with relatively small increases in the ASN, as indicated above. 
Theoretical

Simulated

\begin{tabular}{rrrlllll}
\multicolumn{1}{c}{$\mu_{1}$} & \multicolumn{1}{c}{$\mu$} & ASN & \multicolumn{1}{c}{$Q$} & \multicolumn{1}{c}{$P$} & ASN & $Q$ & $P$ \\
\hline 28 & -5.000 & 10.39 & .9999 & .0001 & 11.34 & 1.000 & 0.000 \\
& 0.000 & 14.08 & .999 & .001 & 15.38 & 1.000 & 0.000 \\
& 5.000 & 21.43 & .988 & .012 & 20.76 & 0.939 & 0.061 \\
& 10.000 & 37.32 & .578 & .122 & 34.45 & 0.850 & 0.150 \\
14.000 & 48.69 & .50 & .50 & 42.50 & 0.50 & 0.50 \\
& 18.000 & 37.32 & .122 & .878 & 36.74 & 0.158 & 0.842 \\
& 23.000 & 21.43 & .012 & .988 & 23.20 & 0.033 & 0.967 \\
& 28.000 & 14.08 & .001 & .999 & 15.47 & 0.000 & 1.000 \\
& 33.000 & 10.39 & .0001 & .9999 & 11.78 & 0.000 & 1.000 \\
& & & & & & & \\
14 & -5.000 & 32.91 & .99999 & .00001 & 34.05 & 1.000 & 0.000 \\
& 0.000 & 56.31 & .999 & .001 & 57.67 & 1.000 & 0.000 \\
& 5.000 & 149.27 & .878 & .122 & 86.00 & 0.750 & 0.250 \\
& 7.000 & 194.85 & .50 & .50 & 93.17 & 0.50 & 0.50 \\
& 10.000 & 118.67 & .049 & .951 & 99.57 & 0.143 & $0.85 i$ \\
14.000 & 56.31 & .001 & .999 & 58.55 & 0.000 & 1.000 \\
& 19.000 & 32.91 & .00001 & .99999 & 34.80 & 0.000 & 1.000 \\
& & & & & & & \\
6 & -3.000 & 153.58 & .999999 & .000001 & 123.00 & 1.000 & 0.000 \\
& 0.000 & 306.55 & .999 & .001 & 166.00 & 1.000 & 0.000 \\
& 3.000 & 1060.75 & .50 & .50 & 279.50 & 0.50 & 0.50 \\
& 6.000 & 306.55 & .001 & .999 & 300.00 & 0.000 & 1.000 \\
& 9.000 & 153.58 & .000001 & .999999 & 151.50 & 0.000 & 1.000
\end{tabular}

ASN, OC and power function of the SPRT.

Table If 


\section{is Conclusions}

The SPRT has been chosen as the method of selecting an hypothesis, because it has been mathematically proven to possess many properties desired by the statistician and has been shown to perform very well in the analysis of DN-signal data. Future goals of the work presented herein include:

- Mathematical investigation of optimum methods for selecting $\mu_{1}$, and a determination of the factors, which cause the maximum simulated ASN to be more significantly less than the maximum theoretical ASN as $\mu_{1}$ approaches $\mu_{0}$.

- Further mathematical investigation of the relationship between various variables and properties of the SPRT, ie. $\alpha, \beta, A S N$, $\mathrm{OC}$, power and robustness. Extensive testing with newly available DN-signal data will be taken during breached-fuel operation under a variety of plant conditions. The 700 data values employed in the investigations to date were obtaired during a brief steady-state exposed-fuel test conducted in EBRII in May, 1988. (See Figure 3.) Plant modifications are currently underway (Spring, 1989) to install new high-sensitivity 

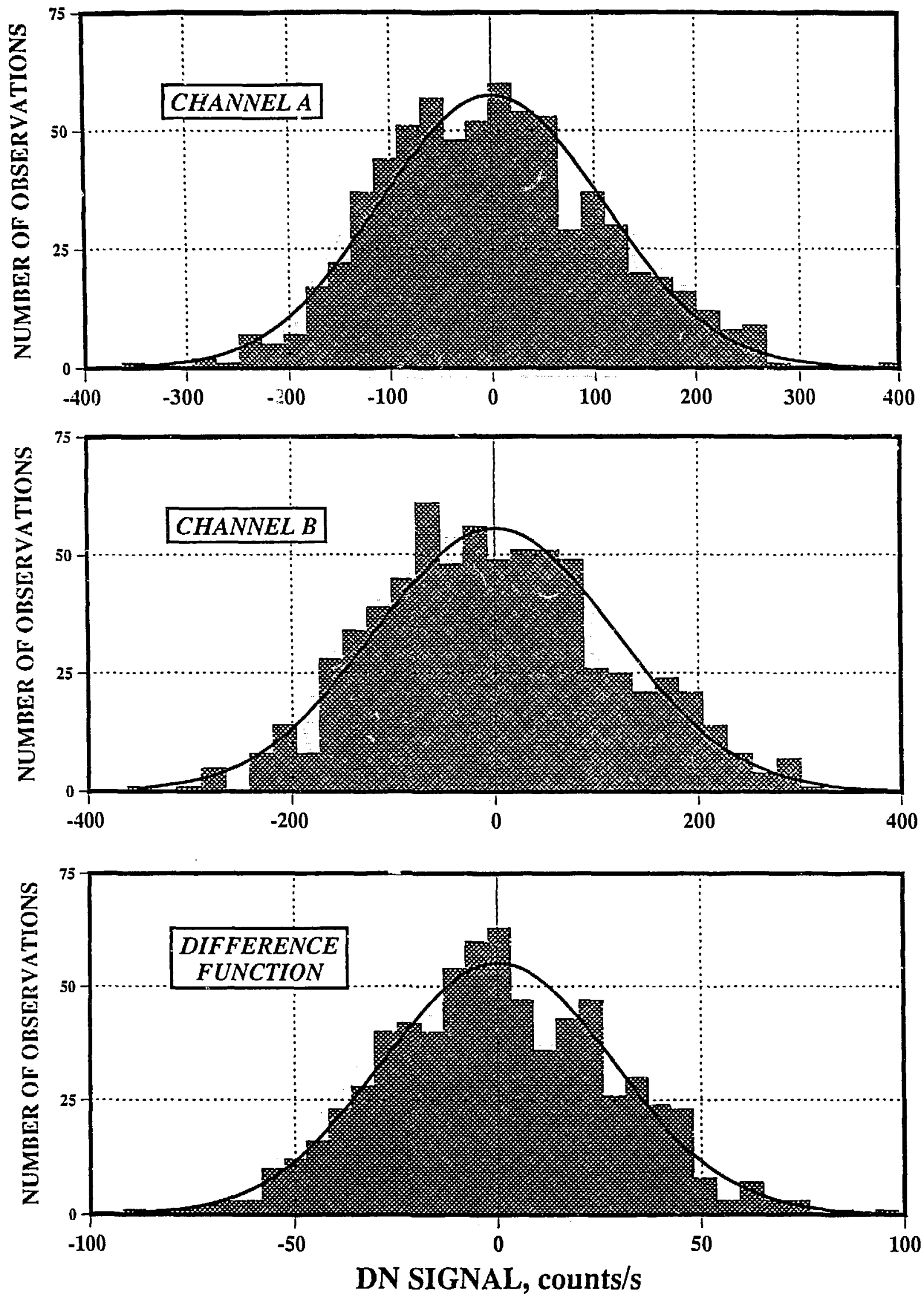

Figure 3. Frequency functions for $X_{1}, X_{2}$ and $X_{k}$, computed from actual experimental DN signals. 
DN detectors (DNDs). The new DND configuration will permit acquisition of DN-signal data from a series of breached-fue! tests that will be conducted under a variety of steady-state, transient-power and transient-flow conditions. This extensive experimental database will provide reliable values of the ASN, $\mathrm{OC}$ and power functions and enal: le the authors to fully explore the quality of the SPRT and discrepancies between theoreticil and simulated values under all breached-fuel conditions anticipated during normal operation of an LMR.

- Research on the generalized truncated SPRT (GSPRT) to reduce the ASN [4]. This SPRT uses 'variable stopping boundaries' with the goal of enabling it to reach a decision faster when $\mu$ is close to $\left(\mu_{0}+\mu_{1}\right) / 2$. This advantage of the GSPRT has been shown to become more pronounced as the error probabilities become smaller [14]. The authors ultimately desire to combine the generalized truncated SPRT and the standard SPRT and to utilize the strong points of each to result in a faster decision. Research on the GSPRT is currently underway and results are encouraging. 
- Research on clipped SPRT's and moving window SPRT's. These SPRT's do not allow the ASN to exceed some prespecified value. This is done by simply beginning the SPRT again (clipped SPRT) or by changing the function of observations in the critical inequality (moving window SPRT) in some mathematically convenient way.

- Application of the SPRT to more than two detectors.

- Encoding the final system in FORTRAN and implementing it as part of the expert system described in the introduction of this paper.

- Extension of the SPRT from analysis of DN-signal data, specifically, to general signal validation and sensor operability applications for all areas of nuclear reactor operation wherein a given physical variable is being monitored by multiple, redundant sensors (e.g. thermocouples, pressure transducers, flux monitors, flow meters, etc.). 


\section{References}

[1] Wald, A., Sequential Analysis, John Wiley \& Sons, New York; 1947.

[2] Wald, A. and Wolfowitz, J., Optimum Character of the Sequential Probability Ratio Test, Annals of Mathematical Statistics, 1948.

[3] Fu, K. S., Sequential Methods in Pattern Recognition and Machine Learning, Academic Press, New York, 1968.

[4] Ghosh, B. K., Sequential Tests of Statistical Hypotheses, Addison-Wesley- Publishing Company: Reading, Massachusetts. 1970.

[5] Hussain, A. B. S., Compound Sequential Probability Ratio Test for the Classification of Statistically Dependent Patterns, IEEE Transactions on Computers, April, 1974.

[6] Dimitriadis, B. and Kazakos, D., A Nonparametric Sequential Test for Data with Markov Dependence, IEEE Transactions on Aerospace and Electronic Systems, May, 1983. 
[7] Tantaratana, S. and Thomas, J. B., Relative Efficiency of the Sequential Probability Ratio Test in Signal Detection, IEEE Transactions on Information Theory, January, 1978.

[8] Schafer, R. E. and Takenaga, R., A Sequential Probability Ratio Test for Availability, Technometrics, February, 1972.

[9] Vesely, W. E., Niyogi, P. K., Lancaster, L. E. and Goldberg, F. F., SPRT Approaches for Assessing Risk Implications of Component Failure Rate Data, Proceedings of the 19S2 DOE Statistical Symposium, Idaho Falls, Idaho, CONF-821021, October, 1982.

[10] Halteman, E. J., Comparing Two Poisson Populations Sequentially: An Application, Rockwell International Report RFP$3878,1986$.

[11] Fehlau, P. E., Pratt, J. C., Markin, J. T. and Scurry Jr., T., Smarter Radiation Monitors for Safeguards and Security, Proceedings of the 24th Annual Meeting of the Institute of Nuclear Materials Management, Vail, Colorado, July 10-13, 1983, Pp. $122-128$. 
[12] Gross, K. C., Expert System for Surveillance and Diagnosis of Breached Fuel Elements, Argonne National Laboratory, U.S.

Patent Number 4,803,040, February, 1989.

[13] Shapiro, S. S. and Wilk, M. B., An Analysis of Variance Test for Normality (Complete Sampies), Biometrika, December. 1965.

[14] Tantaratana, S. and Poor, H. V., Asymptotic Efficiencies of Truncated Sequential Tests, IEEE Transactions on Information Theory, November, 1982. 